\title{
Safety of Anthroposophic Medicinal Products: An Analysis of Adverse Drug Reactions from German Pharmacovigilance Databases
}

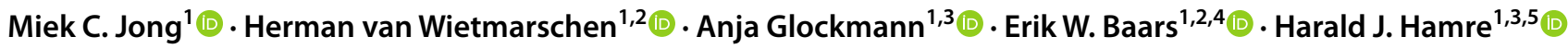

Accepted: 14 May 2021 / Published online: 28 July 2021

(c) The Author(s) 2021

\begin{abstract}
Background Anthroposophic medicinal products (AMPs) are widely used in Europe and world-wide.

Objective To determine the frequency of reported adverse drug reactions (ADRs) from all AMPs on the market, in absolute numbers and relative to the maximum daily administration doses (MDADs).

Patients and Methods Retrolective safety analysis of AMP-related ADRs in pharmacovigilance databases of four AMP Marketing Authorisation Holders in Germany. For each ADR, information about the patient, outcome, causality and AMP was retrieved. Primary outcome was the frequency of reported ADRs relative to MDADs sold.

Results In the period 2010-2017, a total of 5506 ADRs were reported that had occurred in 2765 different patients, comprising 370 different AMPs. A total of 104 ADRs (1.9\%) were classified as serious. The frequency of ADRs for all AMPs was 1.50 per million MDADs. For serious ADRs the frequency was 0.03 per million MDADs. ADRs were more frequently reported with parenteral AMP administration (injections) than with oral or local administration (18.85 vs. 0.59 vs. 1.61 ADRs per million MDADs, respectively; $p<0.0001)$. The large majority of users $(91.9 \%)$ had recovered or were recovering from the ADRs and there were no reports with a fatal outcome. Most frequently reported ADR symptom was injection site inflammation for parenteral AMPs (4.66 ADRs per million MDADs), nausea for oral AMPs (0.03 ADRs per million MDADs), and eye irritation for locally administered AMPs (0.23 ADRs per million MDADs).

Conclusions In this retrolective safety analysis of pharmacovigilance data, the frequency of ADRs to AMPs was 1.50 per million MDADs. Notably, the ADR frequency in this study based on spontaneous reporting is not directly comparable to frequencies in prospective clinical studies nor to frequencies based on other measures of patient exposure than MDADs.
\end{abstract}

Herman van Wietmarschen

h.vanwietmarschen@louisbolk.nl

1 ESCAMP - European Scientific Cooperative on Anthroposophic Medicinal Products, Zechenweg 6, 79111 Freiburg, Germany

2 Louis Bolk Institute, Hoofdstraat 24, 3972 LA Driebergen, The Netherlands

3 Institute for Applied Epistemology and Medical Methodology at the Witten/Herdecke University, Zechenweg 6, 79111 Freiburg, Germany

4 University of Applied Sciences Leiden, Zernikedreef 11, 2333 CK Leiden, The Netherlands

5 Faculty of Health, Witten/Herdecke University, Gerhard-Kienle-Weg 4, 58313 Herdecke, Germany

\section{Key Points}

This retrolective safety analysis of pharmacovigilance data covered 99.4\% (1596/1606) of all AMPs marketed in Germany during the study period 2010-2017.

A total of 5506 ADRs were identified that had occurred in 2765 different patients, comprising 370 different AMPs.

The relative frequency of ADRs for AMPs was 1.5 per million maximum daily administration doses sold.

The large majority of patients $(91.9 \%)$ had recovered or were recovering from the ADRs and there were no reports with a fatal outcome. 


\section{Introduction}

\subsection{Anthroposophic Medicinal Products (AMPs)}

Anthroposophic medicine (AM) is an integrative whole medical system involving special medications (anthroposophic medicinal products, AMPs) and AM non-medication therapies $[1,2]$ in addition to conventional therapy as needed. AMPs were first introduced in Europe in the 1920s and are now used in more than 60 other countries world-wide, including 20 European countries [3]. AMPs are manufactured from substances of botanical, chemical, mineral, or zoological origins [4], and can be defined by three essential features [4-6]:

- They are formulated and developed in accordance with the anthroposophic knowledge of man and nature.

- They are manufactured by procedures that are either specifically anthroposophic or common to those of homeopathic medicinal products.

- Their intended use is according to the principles of AM.

Specific anthroposophic manufacturing procedures include the production of metal mirrors (deposits of metals in reduced state onto a surface) by chemical vapor decomposition; the processing of herbs by fermentation, toasting, carbonizing, incineration and digestion (heat treatment at $37{ }^{\circ} \mathrm{C}$ ); and the cultivation of plants in soils pre-treated with diluted metal salts (vegetabilization) [4, 7]. AMPs are manufactured in concentrated form as well as in homeopathic decimal potencies (' $\mathrm{D}$ ', involving successive 1:10 dilutions) and are administered as oral, rectal, vaginal, conjunctival, nasal or percutaneous applications or by subcutaneous, intracutaneous or intravenous injections [4]. Quality standards for starting materials and manufacturing procedures of AMPs are described in the European Pharmacopoeia (Ph.Eur.), in pharmacopoeias issued by German (Deutsches Arzneibuch [DAB], Deutsches Homöopathisches Arzneibuch [HAB]), French (Pharmacopée Francaise, Ph.Fr.) or Swiss (Pharmacopoeia Helvetica, Ph.Helv.) authorities or in the Anthroposophic Pharmaceutical Codex (APC) [4]. All AMPs are manufactured according to Good Manufacturing Practice standards and subject to modern drug regulation. Toxicologically relevant starting materials (e.g. aconite, celandine, cinnabar) are highly diluted according to safety requirements of European regulations [8-10].

In Germany, where this study was conducted, AMPs are marketed according to a special regulatory provision for Complementary and Alternative Medicines (CAM) and Traditional Medicinal Products (TMP) [11] in the German
Medicines Act. In accordance with this provision, experts on AMPs (pharmacy, toxicology, clinical pharmacology, statistics, clinical use) support the work of the Federal Institute for Drugs and Medical Devices (BfArM) in the Commission $\mathrm{C}$ for AMPs [11]. In accordance with the German Medicines Act, marketing authorization holders (MAHs) of AMPs are obliged to comply with the same pharmacovigilance provisions as MAHs of other finished products on the market [12].

AMPs are prescribed by physicians, along with conventional medicinal products as needed, for the whole range of acute and chronic diseases, with a focus on children's diseases [13], family medicine, and particularly chronic diseases necessitating long-time complex treatments [2, 14]. In Germany and Switzerland, AM treatment is integrated with conventional care in a number of inpatient clinics, including two academic teaching hospitals offering tertiary care [2].

The most frequently prescribed AMPs are mistletoe (Viscum album) products, applied as subcutaneous (s.c.) injections as add-on to standard cancer therapy in order to improve quality of life, relieve symptoms, reduce side effects from anticancer therapies or support antitumor control [15]. On the German market, four different brands of mistletoe AMPs are available, manufactured in Germany or Switzerland from Viscum growing on different host trees [15-17]. Some AMPs are sold over the counter (OTC) as self-medication for consumers and patients for minor ailments, such as eye irritation, common cold, sleeplessness and certain skin conditions [18].

In an updated systematic review of clinical studies of AM therapy for any indication, 235 AMP studies of varying design and quality showed predominantly good clinical outcomes and high patient satisfaction [19]. Notably, a number of older AMPs studies with quality problems had been performed by physicians without training in clinical research. Since the turn of the millennium, research into AMP therapy has become increasingly professionalized. Recently, a consensus-based comprehensive research strategy for AM including AMP therapy has been published [20].

\subsection{Safety of AMPs}

In order to minimize possible risks from AMP use, providing adequate information to users is important. Information on correct use of AMPs and related safety issues is provided in person by physicians prescribing AMPs and pharmacists dispensing them, and in the AMP patient information leaflets. Therein, AMP users are informed about contraindications, interactions with other medicinal products, use by pregnant or breast-feeding women, known side effects (i.e. adverse drug reactions, ADRs) and precautions against these. Upon occurrence of ADRs, including reactions not listed in the leaflet, users are recommended to seek further advice from 
their physician or pharmacist as needed. In Germany, AMP users may also report side effects directly to the Federal Institute for Drugs and Medical Devices.

ADRs to AMPs have been assessed in prospective clinical studies and systematic reviews thereof [7, 19, 21-25], and in an analysis of pharmacovigilance data on AMP injectables by Jong et al. [26]. In these studies, ADRs to AMPs were rare and mostly of mild-to-moderate intensity [7, 19, 21-26].

The European Scientific Cooperative on Anthroposophic Medicinal Products (ESCAMP) is pursuing a research strategy that includes three analyses of ADRs to the entire group of AMPs: a prospective multicenter pharmacovigilance study of 44,662 patients with 311,731 AMP prescriptions (EvaMed [7]), a planned systematic review of ADRs in prospective clinical AMP studies, and the present analysis of ADR reports in pharmacovigilance databases of MAH of AMPs. All three analyses will assess the frequency and characteristics of ADRs for the entire group of AMPs and in subgroups thereof, allowing for comparisons across the three datasets.

The present analysis builds on the work of Jong et al. [26], both being retrolective analyses of ADR datasets from MAHs, and both including AMPs. While Jong et al. analyzed AMPs together with homeopathic products, the present analysis is limited to AMPs only. On the other hand, the present analysis covers a wider scope of AMPs than Jong et al. (any pharmaceutical form vs. injectables only) as well as a more recent time period (2010-2017 vs. 2007-2009). Since 2009, pharmacovigilance procedures have improved considerably due to adoption $[27,28]$ and implementation $[29,30]$ of legal obligations and new guidelines for pharmacovigilance in Europe. Thus, the present analysis covers ADRs to all AMPs under recent pharmacovigilance conditions.

\section{Methods}

\subsection{Objectives}

The primary objective of this study was to determine the frequency of reported ADRs from all AMPs on the market, in absolute numbers and relative to the maximum daily administration doses (MDADs) of AMPs sold. Other objectives were:

- To describe the characteristics of AMP-related ADRs;

- To determine the ADR frequency in subgroups according to patient and AMP characteristics;

- To compare the relative ADR frequency between different AMP administration routes, concentrations and starting materials;

- To describe the most frequently reported ADRs.

\subsection{Design and Setting}

This study was a retrolective analysis of AMP-related ADRs from German pharmacovigilance databases that were reported in the period 2010-2017. Five MAHs of AMPs were invited to participate, of which four agreed to participate: Helixor Heilmittel GmbH (Rosenfeld, Germany), Iscador (Arlesheim, Switzerland), WALA Heilmittel GmbH (Bad Boll/Eckwälden, Germany), and Weleda AG (Arlesheim, Switzerland). The pharmacovigilance data from the four participating MAHs covered $99.4 \%$ $(1596 / 1606)$ of all AMPs marketed in Germany in the period 2010-2017.

\subsection{Inclusion and Exclusion Criteria for Adverse Drug Reactions (ADRs)}

ADRs were included in this analysis if they fulfilled all of the following criteria:

1. Filed by the MAH in the period 1 January 2010 to 31 December 2017.

2. Related to the use of an AMP that was prepared according to the APC [4].

3. Had occurred in humans.

4. Related to the use of an AMP that was sold in Germany.

5. Causal relationship to the AMP in question was assessed by the Qualified Person for PharmacoVigilance (QPPV) of the MAH as one of the following:

5.1. Certain

5.2. Probable/likely

5.3. Possible

5.4. Not assessable

5.5. Not applicable:

5.5.1. ADR from incorrect use of the AMP

5.5.2. ADR reported in terms of lack of effect or similar

5.5.3. ADR causally linked to another AMP that was concomitantly administered in that patient.

ADRs for which the causal relationship to AMP use had been assessed by the QPPV of the MAH as 'unlikely/ remote' or 'not related' were excluded from the analysis.

\subsection{Outcome Measures}

ADR-related outcomes analysed were:

a. Date of report of the ADR case. 
b. Type of report (i.e. spontaneous, literature, clinical study).

c. Qualification of the reporter (i.e. consumer/patient, healthcare professional, non- healthcare professional).

d. Description of the ADR, according to the Medical Dictionary for Regulatory Activities (MedDRA).

e. Seriousness of the ADR, according to the MAH.

f. Labelling information of the ADR (in the patient information leaflet), at the time of entry to the database.

g. Outcome of the ADR, according to the reporter.

h. Causality of the ADR, according to the MAH (certain, probable/likely, possible, not assessable, not applicable).

AMP-related outcomes analysed were:

i. Name of the AMP.

j. Name of the MAH of the AMP.

k. Route of administration of the AMP.

1. Pharmaceutical dose form of the AMP.

m. Number of starting materials of the AMP.

n. Dilution(s) of the active substance(s) of the AMP.

o. Origin of starting materials of the AMP.

Patient-related outcomes analysed were:

p. Gender.

q. Age of the patient at ADR occurrence.

Of the analysed outcomes, outcomes g, i, k, l, p, q had been submitted to the MAH by consumers/patients, healthcare professionals and other reporters; outcomes a, b, c, d, $\mathrm{e}, \mathrm{f}, \mathrm{h}$ were added/coded by the MAH; and outcomes $\mathrm{j}, \mathrm{m}$, $\mathrm{n}$, o were coded by the authors (AG, MJ). For ADRs with a causal relationship assessed as not applicable (inclusion criterion 5.5, above), some or all of outcomes e-g had not been routinely assessed by the MAH and were therefore not analysed.

The primary outcome was the frequency of overall reported AMP-related ADRs in the period 2010-2017, relative to the total sales volume of AMPs on the market in the same period. The total sales volume was calculated as the absolute number of MDADs sold. Secondary outcomes were the absolute and relative frequencies of ADRs according to outcome items $\mathrm{b}$ through $\mathrm{q}$.

\subsection{Definitions, Data Categorization/Classification and Data Preparation}

Serious adverse events were defined as adverse events that result in death, are life-threatening, require inpatient hospitalization or prolongation of existing hospitalization, result in persistent or significant disability/incapacity, are a congenital anomaly/birth defect, or are another medically important condition [31].

Age groups were categorized according to guidelines on good pharmacovigilance practice of the European Medicines Agency [32]. Causal relationship between the adverse event and AMP administration was categorized according to the WHO Uppsala Monitoring Centre guidelines as certain, probable/likely, possible, unlikely/remote, not assessable [33] with the additional categories not applicable, and not related.

Reported ADRs were classified according to MedDRA [34] and presented on the Preferred Term (PT) and System Organ Class (SOC) levels. For interpretation of findings, the relative frequency of ADRs was classified into very common $(\geq 10 \%)$, common $(1 \%<\chi<10 \%)$, uncommon $(0.1 \%<\chi<1 \%)$, rare $(0.01 \%<\chi<0.1 \%)$, and very rare $(<0.01 \%)$ [35].

In order to guarantee that the provided data could not be linked to identifiers of individual patients, AMP MAHs provided a de-identified dataset for this analysis, in accordance with general data protection regulations [36]. The dataset was collected in an Excel document with predefined fields sent to the companies. All ADR-related characteristics extracted were coded by one author (AG) according to a predefined code book. The coding was independently cross-checked by another author (MJ), in order to identify and correct possible errors or omissions. Sales volumes of 1596 AMPs (in kilos, litres or other unit) in the years 2010-2017 were converted by two authors (MJ, HvW) to absolute numbers of MDADs sold, which was regarded to be an approximate estimation of MDADs administered. MDADs were calculated according to the approved MDAD of the individual AMP as listed in the pharmaceutical catalogues of the respective MAH. The MDAD per gender and age group was estimated from the proportion between the respective AMP user groups in a prospective pharmacovigilance study of 44,662 patients prescribed 311,731 AMPs in Germany [7].

\subsection{Statistics, Post Hoc Analyses}

Descriptive analyses were carried out in SPSS Version 24, according to a predefined statistical analysis plan. Numbers and frequencies of ADRs related to outcomes b through $q$ were calculated, and differences between categories were assessed using the Chi-square test. All $p$-values are descriptive, without accommodation for multiple hypothesis testing.

Three post hoc analyses were performed:

- The frequency of ADRs per MDAD for parenteral AMPs was compared to corresponding data from two 
previous safety studies with available datasets $[7,26]$ for which data had been collected in 2007-2009 [26] and 2001-2010 (EvaMed [7]), respectively. For these two studies MDADs for parenteral AMPs were calculated by the authors ( $\mathrm{HvW}, \mathrm{AG})$.

- Comparison of AMPs with reported ADRs in this analysis and in the EvaMed study [7].

- Description of ADRs classified as SOC Hepatobiliary disorders.

\section{Results}

\subsection{ADRs: Frequencies and Characteristics (Table 1)}

In the period 2010-2017, a total of 5506 ADRs fulfilling all inclusion criteria were identified in the pharmacovigilance databases of the participating AMP MAHs and included in the analysis. The primary outcome, frequency of reported AMP-related ADRs relative to the MDAD of AMPs sold, was 1.5 ADR per million MDADs, which was classified as very rare. Of the 5506 ADRs, $1.9 \%(n=104)$ were serious ADRs. The overall frequency of reported serious ADRs was 0.03 ADR per million MDADs, classified as very rare. As shown in Table 1, in most cases the causal relationship between ADRs and AMP administration was assessed/classified as 'possible' (67.4\%, $n=3711 / 5506$ ) and the outcome of the ADR was 'recovered/resolved' ( $67.8 \%, n=3732 / 5506)$ upon reporting. There were no reports of ADRs with a fatal outcome. Most $(60.7 \%, n=3342 / 5506)$ ADRs were already mentioned (labelling of safety information) in the patient information leaflet of the respective AMP. The unlabelled ADRs were three times more likely to be serious ADRs than the labelled ADRs $(3.1 \%, n=57 / 1850$ vs. $1.2 \%, n=41 / 3341$, $p<0.0001)$. Recovery from the ADRs was similar for the unlabelled $(87.7 \%, n=1291 / 1472)$ and labelled $(85.5 \%$, $n=2282 / 2669)$ ADRs. Further data on labelled versus unlabelled ADRs are presented in Table S1 (Online Supplementary Material).

Data on ADR reports excluded from the analysis were provided by two of the four participating MAHs (the MAHs were not obliged to provide these data). These two MAHs together had identified $n=3330$ ADR reports related to AMP use in humans in Germany, of which 612 reports were excluded because the causal relationship to the AMP in question had been assessed by the QPPV of the MAH to be unlikely/remote $(14.1 \%, n=465 / 3330$ reports) or not related $(4.4 \%, n=147)$, while $81.6 \%$ $(n=2718)$ of the ADR reports were included.
Table 1 Characteristics of adverse drug reactions (ADRs)

\begin{tabular}{|c|c|c|c|}
\hline \multirow[t]{2}{*}{ Characteristic } & \multicolumn{2}{|c|}{ ADRs } & \multirow[t]{2}{*}{ ADRs/MDADs } \\
\hline & $N$ & $\%$ & \\
\hline \multicolumn{4}{|l|}{ Seriousness } \\
\hline Non-serious ADRs & 5331 & 96.8 & 1.45 \\
\hline Serious ADRs & 104 & 1.9 & 0.03 \\
\hline Not applicable ${ }^{\mathrm{b}}$ & 71 & 1.3 & 0.02 \\
\hline Total & 5506 & 100.0 & 1.50 \\
\hline \multicolumn{4}{|l|}{ Causality } \\
\hline Certain & 615 & 11.2 & 0.17 \\
\hline Probable/likely & 393 & 7.1 & 0.11 \\
\hline Possible & 3711 & 67.4 & 1.01 \\
\hline Not assessable & 679 & 12.3 & 0.18 \\
\hline Not applicable ${ }^{\mathrm{b}}$ & 108 & 2.0 & 0.03 \\
\hline Total & 5506 & 100.0 & 1.50 \\
\hline \multicolumn{4}{|l|}{ Outcome } \\
\hline Recovered/resolved & 3732 & 67.8 & 1.02 \\
\hline Recovering/resolving & 318 & 5.8 & 0.09 \\
\hline Recovered/resolved with sequelae & 13 & 0.2 & 0.00 \\
\hline Sequelae & 1 & 0.0 & 0.00 \\
\hline Not recovered/not resolved & 266 & 4.8 & 0.07 \\
\hline Fatal & 0 & 0.0 & 0.00 \\
\hline Unknown & 1097 & 19.9 & 0.30 \\
\hline Not assessable & 2 & 0.0 & 0.00 \\
\hline Not applicable ${ }^{\mathrm{b}}$ & 77 & 1.4 & 0.02 \\
\hline Total & 5506 & 100.0 & 1.50 \\
\hline \multicolumn{4}{|l|}{ Labelling $^{\mathrm{c}}$} \\
\hline Yes & 3342 & 60.7 & 0.91 \\
\hline No & 1854 & 33.7 & 0.50 \\
\hline Equivalent $^{\mathrm{d}}$ & 127 & 2.3 & 0.03 \\
\hline Not applicable ${ }^{b}$ & 110 & 2.0 & 0.03 \\
\hline Unknown & 52 & 0.9 & 0.01 \\
\hline Not assessed & 20 & 0.4 & 0.01 \\
\hline No approval in & 1 & 0.0 & 0.00 \\
\hline Total & 5506 & 100.0 & 1.50 \\
\hline
\end{tabular}

$M D A D$ s maximum daily administration doses sold, AMP anthroposophic medicinal product

${ }^{\mathrm{a}} \times 1$ million

${ }^{\mathrm{b}}$ Not applicable: incorrect use of the AMP, the ADR was reported in terms of lack of effect or similar, or the ADR was evaluated to be causally linked to another AMP that was concomitantly administered in that patient

${ }^{c}$ Labelling information on the ADR in the patient information leaflet

${ }^{\mathrm{d}}$ Equivalent (similar) to the labelling of the ADR in the patient information leaflet

\subsection{ADR Reporting (Fig. 1, Table 2)}

Of the 5506 ADR reports, $99.1 \%(n=5455)$ were spontaneous reports, $0.7 \%(n=40)$ were extracted from clinical study reports, and $0.2 \%(n=11)$ were identified in other literature. Most ADRs $(51.9 \%, n=2855 / 5506)$ were 
Fig. 1 Number of anthroposophic medicinal product (AMP)-related adverse drug reactions (ADRs) per year and per type of reporter

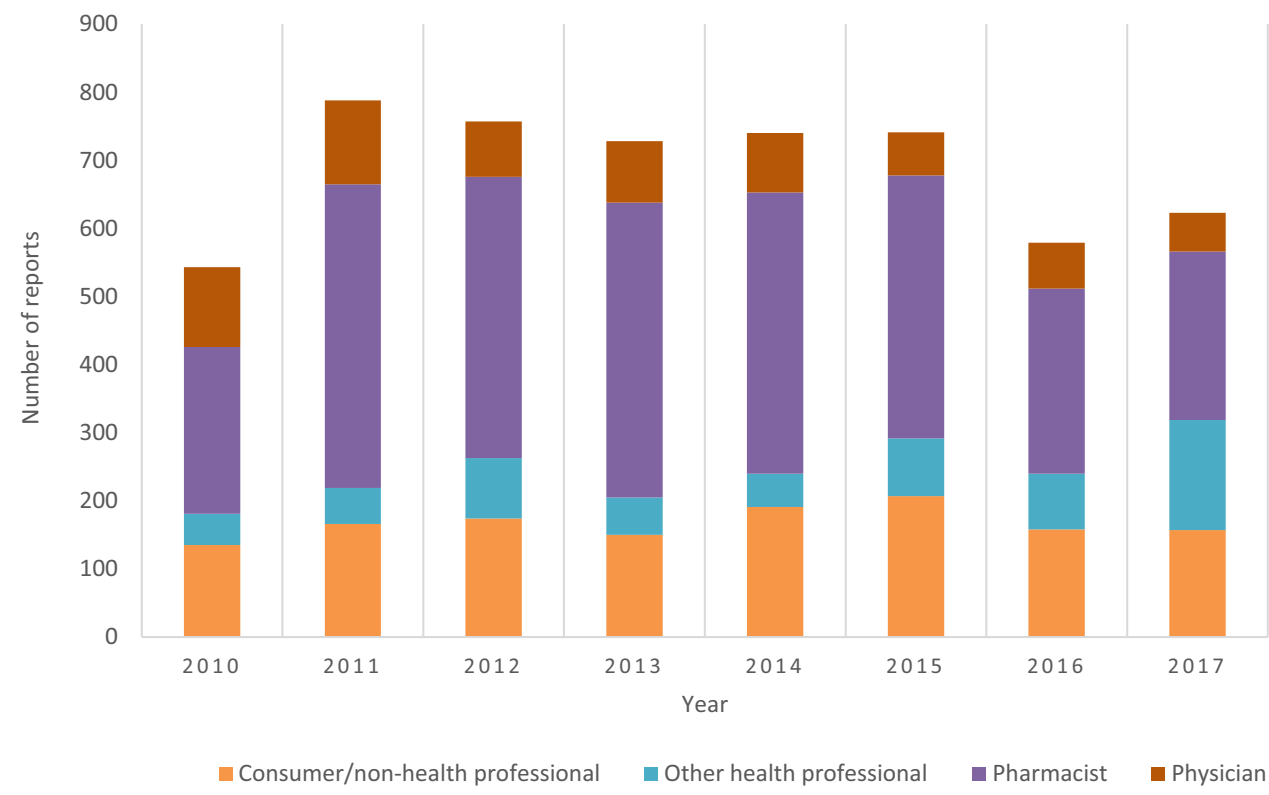

\begin{tabular}{|c|c|c|c|c|c|c|}
\hline \multirow[t]{2}{*}{ Patient characteristic } & \multicolumn{2}{|c|}{$\begin{array}{l}\text { Patients with } \\
\text { ADRs }\end{array}$} & \multicolumn{2}{|c|}{ ADRs } & \multirow{2}{*}{$\begin{array}{l}\text { MDADs }^{\mathrm{a}} \\
N\end{array}$} & \multirow[t]{2}{*}{ ADRs/MDADs ${ }^{\mathrm{a}}$} \\
\hline & $N$ & $\%$ & $N$ & $\%$ & & \\
\hline \multicolumn{7}{|l|}{ Gender } \\
\hline Female & 2236 & 80.9 & 4567 & 82.9 & 2130 & 2.14 \\
\hline Male & 500 & 18.1 & 886 & 16.1 & 1542 & 0.57 \\
\hline Unknown & 29 & 1.0 & 53 & 1.0 & n.a. ${ }^{b}$ & n.a. ${ }^{b}$ \\
\hline Total & 2765 & 100 & 5506 & 100.0 & 3672 & 1.50 \\
\hline \multicolumn{7}{|l|}{ Age group } \\
\hline Neonate (0-27 days) & 17 & 0.6 & 32 & 0.6 & $455^{\mathrm{c}}$ & $0.51^{\mathrm{c}}$ \\
\hline Infant ( 28 days -23 months) & 100 & 3.6 & 200 & 3.6 & & \\
\hline Child (2-11 years) & 141 & 5.1 & 234 & 4.2 & 1642 & 0.14 \\
\hline Adolescent (12-18 years) & 24 & 0.9 & 57 & 1.0 & 202 & 0.28 \\
\hline Adult (19-64 years) & 1477 & 53.4 & 3062 & 55.6 & 981 & 3.12 \\
\hline Elderly ( $\geq 65$ years) & 550 & 19.9 & 1086 & 19.7 & 393 & 2.76 \\
\hline Unknown & 456 & 16.5 & 835 & 15.2 & n.a. ${ }^{b}$ & n.a. ${ }^{b}$ \\
\hline Total & 2765 & 100 & 5506 & 100.0 & 3672 & 1.50 \\
\hline
\end{tabular}

$M D A D s$ maximum daily administration doses sold

${ }^{\mathrm{a}} \times 1$ million

${ }^{\mathrm{b}}$ n.a. not applicable, MDADs and Relative Frequency could not be calculated for unknown gender and age group

${ }^{c}$ MDADs and Relative Frequency for age group $<24$ months reported by pharmacists, followed by consumers or other non-healthcare professionals (24.3\%), physicians (12.5\%) and by other healthcare professionals (11.3\%) (Fig. 1). In the period 2010-2017, the number of AMP-related ADRs reported per year varied between 547 and 788 ADRs (Fig. 1) with an average of 688 ADRs reported per year.

The 5506 reported ADRs had occurred in 2765 patients (Table 2). The relative frequency of ADRs was significantly 
higher in females compared to males ( 2.14 vs. 0.57 per million MDADs, respectively, $p<0.0001$ ), and significantly higher in adults and elderly patients compared to adolescents, children, infants/neonates (3.12 and 2.76 vs. 0.28 , 0.14 and 0.51 per million MDADs, respectively, $p<0.0001$ ) (Table 2).

\subsection{ADR Frequencies Related to AMP Characteristics (Table 3)}

The 5506 ADRs that had occurred in 2010-2017 were caused by 370 different AMPs (23.2\% of all AMPs on the German market in this period, $n=370 / 1596$ ), while the majority of AMPs $(76.8 \%, n=1226)$ were not associated with any ADR. For each of the 370 individual AMPs with ADRs, the relative frequencies were classified as very rare.

As shown in Table 3, the majority of absolute numbers of ADRs was reported with local administration of the AMP $(52.7 \%, n=2900 / 5506)$. However, the relative frequency of ADRs was significantly higher with parenteral AMP administration, compared to local and oral administration (18.85 vs. 1.61 vs. 0.59 ADRs per million MDADs, respectively, $p<0.0001$ ) (Table 3). Among AMPs of different concentrations, the relative ADR frequency ranged from 0.79 to 2.81 ADRs per million MDADs ('D1-D3 dilution' and 'mixture of different dilution categories in one AMP', respectively). Among AMPs with one single starting material, the relative ADR frequency ranged from 0.32 to 2.77 ADRs per million
Table 3 Adverse drug reaction (ADR) frequency related to anthroposophic medicinal product (AMP) characteristics

\begin{tabular}{|c|c|c|c|c|}
\hline \multirow[t]{2}{*}{ AMP characteristic } & \multicolumn{2}{|l|}{ ADRs } & \multirow{2}{*}{$\begin{array}{l}\text { MDADs }^{\mathrm{a}} \\
N\end{array}$} & \multirow[t]{2}{*}{ ADRs/MDADs ${ }^{\mathrm{a}}$} \\
\hline & $N$ & $\%$ & & \\
\hline \multicolumn{5}{|l|}{ Route of administration } \\
\hline Local (cutaneous, ophthalmic, rectal, other) & 2900 & 52.7 & 1802 & 1.61 \\
\hline Parenteral (injectables) & 1539 & 28.0 & 82 & 18.85 \\
\hline Oral & 1060 & 19.3 & 1789 & 0.59 \\
\hline Unknown & 7 & 0.1 & n.a. ${ }^{b}$ & n.a. ${ }^{\text {b }}$ \\
\hline Total & 5506 & 100.0 & 3672 & 1.50 \\
\hline \multicolumn{5}{|l|}{ Concentration } \\
\hline Non-diluted & 3795 & 68.9 & 1907 & $1.99^{\mathrm{c}}$ \\
\hline D1-D3 dilution & 1097 & 19.9 & 1393 & $0.79^{c}$ \\
\hline$\geq \mathrm{D} 4$ dilution & 431 & 7.8 & 310 & $1.39^{\mathrm{c}}$ \\
\hline Other ${ }^{\mathrm{e}}$ & 176 & 3.2 & 63 & $2.81^{\mathrm{c}}$ \\
\hline Unknown & 7 & 0.1 & n.a. ${ }^{b}$ & n.a. ${ }^{\text {b }}$ \\
\hline Total & 5506 & 100.0 & 3672 & 1.50 \\
\hline \multicolumn{5}{|l|}{ Starting material } \\
\hline Botanical & 2680 & 48.7 & 1831 & $1.46^{\mathrm{d}}$ \\
\hline Zoological & 92 & 1.7 & 33 & $2.77^{\mathrm{d}}$ \\
\hline Chemical & 51 & 0.9 & 153 & $0.33^{\mathrm{d}}$ \\
\hline Mineral & 12 & 0.2 & 38 & $0.32^{\mathrm{d}}$ \\
\hline Vegetabilized metal $^{\mathrm{f}}$ & 18 & 0.3 & 8 & $2.13^{\mathrm{d}}$ \\
\hline More than one starting material & 1646 & 29.9 & 872 & $1.89^{\mathrm{d}}$ \\
\hline Compositions & 1004 & 18.2 & 737 & $1.36^{\mathrm{d}}$ \\
\hline Unknown & 3 & 0.1 & n.a. ${ }^{b}$ & n.a. ${ }^{b}$ \\
\hline Total & 5506 & 100.0 & 3672 & 1.50 \\
\hline
\end{tabular}

MDADs maximum daily administration doses sold

${ }^{\mathrm{a}} \times 1$ million

${ }^{\mathrm{b}}$ n.a. not applicable, sales volumes could not be calculated for unknown origin of route of administration, and unknown concentration or starting material

${ }^{\mathrm{c}}$ Calculated as number of ADRs/MDAD per category of AMP concentration

${ }^{\mathrm{d}}$ Calculated as number of ADRs/MDAD per category of starting material

${ }^{\mathrm{e}}$ Mixture of different dilution categories in one AMP

${ }^{\mathrm{f}}$ Herbs cultivated with specially prepared and diluted metals 
MDADs (starting material of mineral and zoological origin, respectively) (Table 3 ).

\subsection{ADR Symptoms Related to Route of Administration, Most Frequent System Organ Class (SOC; Table 4)}

For locally and parentally administered AMPs, most frequently reported ADR symptoms were administration/ application-related, such as local irritation, pain, erythema, pruritus and inflammation. For orally administered AMPs, the most common ADR symptoms were nausea, rash and pruritus (Table 4).

Among ADRs to all AMPs, the most frequent MedDRA SOC groups were general disorders and administration site conditions (34.4\%, $n=1896 / 5506$ ADRs), eye disorders (18.7\%), and skin and subcutaneous tissue disorders (17.8\%) (Table 4).
Table 4 Most frequently reported adverse drug reactions (ADRs) by Preferred Term per route of administration, and System Organ Class

\begin{tabular}{|c|c|c|c|}
\hline \multirow[t]{2}{*}{ MedDRA classification } & \multicolumn{2}{|l|}{ ADR } & \multirow[t]{2}{*}{ ADRs/MDADs } \\
\hline & $N$ & $\%$ & \\
\hline \multicolumn{4}{|l|}{ Preferred term } \\
\hline \multicolumn{4}{|l|}{ Local route of administration } \\
\hline 1. Eye irritation & 409 & 14.1 & $0.23^{\mathrm{a}}$ \\
\hline 2. Application site pain & 279 & 9.6 & $0.15^{\mathrm{a}}$ \\
\hline 3. Application site erythema & 194 & 6.7 & $0.11^{\mathrm{a}}$ \\
\hline 4. Ocular hyperaemia & 176 & 6.1 & $0.10^{\mathrm{a}}$ \\
\hline 5. Application site pruritus & 131 & 4.5 & $0.07^{\mathrm{a}}$ \\
\hline 6. Other & 1711 & 59.0 & $0.95^{\mathrm{a}}$ \\
\hline Total & 2900 & 100.0 & 1.61 \\
\hline \multicolumn{4}{|l|}{ Parenteral route of administration } \\
\hline 1. Injection site inflammation & 380 & 14.6 & $4.66^{\mathrm{a}}$ \\
\hline 2. Injection site pruritus & 52 & 3.4 & $0.64^{\mathrm{a}}$ \\
\hline 3. Injection site erythema & 50 & 3.2 & $0.61^{\mathrm{a}}$ \\
\hline 4. Rash & 49 & 3.2 & $0.60^{\mathrm{a}}$ \\
\hline 5. Injection site nodule & 48 & 3.1 & $0.59^{\mathrm{a}}$ \\
\hline 6. Other & 960 & 62.3 & 11.76 \\
\hline Total & 1539 & 100.0 & 18.85 \\
\hline \multicolumn{4}{|l|}{ Oral route of administration } \\
\hline 1. Nausea & 58 & 5.5 & $0.03^{\mathrm{a}}$ \\
\hline 2. Rash & 50 & 4.7 & $0.03^{\mathrm{a}}$ \\
\hline 3. Pruritus & 44 & 4.2 & $0.02^{\mathrm{a}}$ \\
\hline 4. Abdominal pain & 30 & 2.8 & $0.02^{\mathrm{a}}$ \\
\hline 5. Palpitations & 27 & 2.5 & $0.02^{\mathrm{a}}$ \\
\hline 6. Other & 851 & 80.3 & $0.48^{\mathrm{a}}$ \\
\hline Total & 1060 & 100.0 & 0.59 \\
\hline \multicolumn{4}{|l|}{ System organ class } \\
\hline 1. General disorders and administration site conditions & 1896 & 34.4 & $0.52^{\mathrm{b}}$ \\
\hline 2. Eye disorders & 1030 & 18.7 & $0.28^{\mathrm{b}}$ \\
\hline 3. Skin and subcutaneous tissue disorders & 981 & 17.8 & $0.27^{\mathrm{b}}$ \\
\hline 4. Gastrointestinal disorders & 448 & 8.1 & $0.12^{\mathrm{b}}$ \\
\hline 5. Respiratory, thoracic and mediastinal disorders & 221 & 4.0 & $0.06^{\mathrm{b}}$ \\
\hline 6. Other & 930 & 16.9 & $0.25^{\mathrm{b}}$ \\
\hline Total & 5506 & 100.0 & 1.50 \\
\hline
\end{tabular}

MDADs maximum daily administration doses sold

${ }^{\mathrm{a}}$ Calculated as number of ADRs/MDAD per route of administration $\times 1$ million

${ }^{\mathrm{b}}$ Calculated as number of ADRs/total MDAD $\times 1$ million 


\subsection{Comparison of ADR Frequencies for Parenteral AMPs with Other Studies (Table 5)}

The relative frequencies of ADRs per MDAD for parenteral AMPs in the present study from Germany was compared with two other AMP safety analyses, also from Germany $[7,26]$. In all three studies, ADRs to AMP injectables were very rare $(<0.01 \%$ of MDAD). Compared to the Jong et al. analysis from 2012, also based on MAHs' pharmacovigilance data [26], the relative ADR frequency in the present study was in the same range (Table 5). In the EvaMed study with 9983 patients prescribed AMP injectables [7], the relative ADR frequency was four times higher than in the present study (Table 5).

\subsection{Comparison of AMPs with Reported ADRs in This Analysis and in the EvaMed Study}

While the 5506 ADRs analyzed in this study were caused by 370 different AMPs, 100 ADRs in the EvaMed study [7] were caused by 83 different AMPs. Of the 370 AMPs with
ADRs in this study, $87.8 \%(n=325 / 370)$ that had caused $70 \%(n=3853 / 5506)$ of the ADRs, had not been associated with ADRs in EvaMed.

\subsection{ADRs Classified as SOC Hepatobiliary Disorders}

Five ADRs with altogether eight PTs, occurring in five different patients, had been classified by the respective MAH as SOC Hepatobiliary disorders. The causal relationship between the ADRs had been classified as probable/likely (Patient \#1 in Table 6) and not assessable (Patients \#2-5 with altogether seven PTs).

These five cases were post hoc reassessed with respect to the possible occurrence of herb-induced liver injury (HILI) [37]. The assessment was based on the outcome variables a-o (cf. Sect. 2.4, available for Patients \#1-5), additional free-text information from MAH (Patients \#1-2) and available documentation from a study published by two of the authors (HJH, AG) [22] (Patient \#5). In none of the patients were serum levels of alanine aminotransferase or alkaline phosphatase available, thus initial screening for HILI according to the liver-specific RUCAM algorithm [38] was not possible. Furthermore, no data were available
Table 5 Comparison of adverse drug reaction (ADR) frequencies for parenteral anthroposophic medicinal product (AMP) with other studies

\begin{tabular}{llllll}
\hline Study & Design & Country, period & ADRs & \multicolumn{2}{c}{ ADRs/MDADs $^{\text {a }}$} \\
\cline { 5 - 6 } & & & $N$ & MDAD $^{\mathrm{a}}$ & \\
\hline This study & PV database & DE, 2010-2017 & 1539 & 82.63 & 18.85 \\
Jong et al. [26] & PV database & DE, 2007-2009 & 172 & 6.89 & 24.98 \\
Hamre (EvaMed) [7] & Prospective cohort & DE, 2001-2010 & 28 & 0.37 & 75.65 \\
\hline
\end{tabular}

$P V$ pharmacovigilance, $D E$ Germany, $M D A D s$ maximum daily administration doses sold ${ }^{\mathrm{a}} \times 1$ million

Table 6 Patients with reported adverse drug reactions (ADRs) classified as hepatobiliary disorders: reassessment by the authors

\begin{tabular}{|c|c|c|c|c|c|c|}
\hline Patient \# & Preferred term & SOC: hepatobiliary disorder? & Serious? & $\begin{array}{l}\text { AMP substance with } \\
\text { known hepatotoxic } \\
\text { potential? }\end{array}$ & Outcome & Causality \\
\hline 1 & Administration related reaction & $\begin{array}{l}\text { No: General disorders and } \\
\text { administration site condi- } \\
\text { tions }\end{array}$ & No & No & Resolved & Probable/likely \\
\hline 2 & Hepatic function abnormal & Yes & No & No & Unknown & Unlikely/remote \\
\hline \multirow[t]{2}{*}{3} & Biliary colic & Yes & No & No & Resolved & Not assessable \\
\hline & Liver disorder & Yes & No & & Resolved & Not assessable \\
\hline \multirow[t]{3}{*}{4} & Autoimmune hepatitis & Yes & Yes & Chelidonium majus & Resolved & Unlikely/remote \\
\hline & Drug-induced liver injury & Yes & No & & Resolved & Not assessable \\
\hline & Jaundice & Yes & No & & Resolved & Not assessable \\
\hline 5 & Flatulence & No: Gastrointestinal disorders & No & Chelidonium majus & Resolved & Possible \\
\hline
\end{tabular}

Text in Italics: Classification by authors differs from that of the marketing authorization holder (MAH)

SOC System Organ Class 
on comedications (Patients \#3-4), the temporal relationship between the reported ADR and the AMP in question (Patients \#2-4), comorbidity (Patients \#3-4), or alcohol use (Patients \#1-4).

Patients were aged 48-86 years, and consisted of four females and one male. In Patients 1 and 5 the ADR was not a hepatobiliary disorder. In Patient 2 , the causality was classified as unlikely/remote (most likely cause of the reported ADR was progression of end-stage cancer). In Patient 3 with PTs biliary colic and liver disorder that both resolved, HILI seems unlikely; furthermore, the AMP in question contained no substance with a known hepatotoxic potential and there was no information on comedications, comorbidity or alcohol use.

Patient 4, a 74-year-old woman, had three PTs: autoimmune hepatitis (serious), drug-induced liver injury, and jaundice (both non-serious), all of which resolved. The ADR data were based on a spontaneous report submitted to the MAH by the consumer or other non-health professional. For our reassessment, no data were available on any of the following seven items: the temporal relationship between the ADR and the AMP therapy, nor on the relationship between the PTs autoimmune hepatitis and drug-induced liver injury (whether they were understood as different events or alternative explanations for the same event), likewise there were no data on liver enzyme levels, the diagnostic evidence for autoimmune hepatitis, further comorbidities, comedications, or alcohol use. The presence of autoimmune hepatitis would make HILI less likely [38].

Notably, the AMP in question contains Chelidonium majus (CM), which may cause idiosyncratic HILI in some patients $[39,40]$. Since 2008, for CM-containing products for oral use on the German market with an estimated daily dose $\geq 2.5 \mu \mathrm{g}$ alkaloids calculated as chelidonine, the summary of product characteristics and patient information leaflet have to include certain specified information [41]. Amongst others, the products are contraindicated in subjects with previous or ongoing liver disease or with concomitant intake of other products with liver-damaging properties. When used for more than 4 weeks, serum liver enzyme levels should be controlled [41]. The AMP used by Patient 4 falls into this category. However, in assessments of possible HILI from CM, autoimmune hepatitis is used as the exclusion criterion [39, 40, 42]. Therefore, HILI from CM in Patient 4 seems less likely. Furthermore, without data on serum alanine aminotransferase and alkaline phosphatase, HILI and drug-induced liver injury cannot be reliably diagnosed [43].

In the prospective EvaMed pharmacovigilance study of 311,731 AMP prescriptions, of which 2772 prescriptions were for CM-containing AMPs, no hepatobiliary ADRs occurred [7].

\section{Discussion}

\subsection{Main Findings}

To the best of our knowledge, this is the first study that systematically evaluated all AMP-related ADRs from pharmacovigilance databases after the new EU pharmacovigilance legislation came into place in 2012 [27-30]. Over an 8-year period (2010-2017), an average of 688 AMP therapy-related ADRs per year were collected, in total 5506 ADRs related to 370 different AMPs in 2765 patients. The majority of AMPs on the market in that period $(76.8 \%)$ were not associated with any ADR. The frequency of all AMP-related reported ADRs and all serious ADRs was 1.5 and 0.03 per million MDADs, respectively. At least two-thirds of ADRs were resolved upon reporting, and there were no reports of AMP therapyrelated ADRs with a fatal outcome.

\subsection{Strengths and Limitations}

Strengths of this retrolective safety analysis include a relatively large pharmacovigilance data set of more than 5000 ADRs, a long documentation period of 8 years with the inclusion of almost all AMPs (99.4\%) marketed in Germany, good quality of ADR reports enabling causality assessment in $85 \%$ of cases, and analysis and publication independently of AMP MAHs. Compared to the large EvaMed pharmacovigilance study with more than 300,000 AMP prescriptions [7], this analysis covered 4.5 times more AMPs associated with ADRs.

Several limitations have to be taken into account when interpreting the outcome of this pharmacovigilance analysis. A well-known limitation of spontaneously reported safety data is under-reporting of ADRs [44, 45]. This issue is discussed in section 4.3 'Comparison with Other Studies'. Another limitation was that the primary outcome, relative frequency of overall AMP-related reported ADRs, was calculated using the MDADs sold. Although in the present analysis the MDADs sold was the best possible estimate of the number of administration doses administered, it can be assumed that not all MDADs sold were actually used. Furthermore, relative ADR frequencies with MDADs as denominator are not directly comparable to frequencies with other denominators; for example in the EvaMed study [7] the relative frequency of ADRs to AMP injectables was $0.008 \%$ of MDADs (very rare) and $0.165 \%$ of prescriptions (rare).

In this study, causality of reported ADRs was assessed and labelled as such by the QPPV from the respective $\mathrm{MAH}$, which is in accordance with guidelines of the 
European Medicines Agency [36] and current practice $[46,47]$. Notably, this assessment could differ from the opinion of the reporting consumer or (non-) healthcare professional. Also, as the analysis of ADRs classified as hepatobiliary disorders illustrates, for a proportion of ADR reports submitted to MAHs (in this dataset 15\%), the causal relationship between ADR and product cannot be assessed.

\subsection{Comparison with Other Studies}

The relative frequency of ADRs to parenteral AMPs in the present analysis of data from 2010-2017 was similar to the ADR frequency in a parenteral AMP pharmacovigilance dataset from 2007-2009 [26]. Implementation of the updated EU pharmacovigilance legislation in 2012 [30] has thus not been followed by any apparent changes in the spontaneous reporting rate of AMP-related ADRs to MAHs in Germany. A possible explanation for spontaneous ADR reporting remaining stable is that any improvement in reporting intended with the new EU legislation had already been implemented in Germany: In 2007, shortly before the European pharmacovigilance guidelines Volume 9a were published [48], MAHs in Germany were already systematically collecting and analysing spontaneously reported AMPrelated ADRs.

In contrast to the analysis of spontaneously reported AMP-related ADRs, the EvaMed study was a prospective pharmacovigilance study in which AMPs were prescribed and monitored by physicians at subsequent consultations for possible occurrence of ADRs [7]. The fourfold higher frequency of ADRs to parenteral AMPs in the EvaMed study than in the present study suggests that AMP-related ADRs were under-reported in this safety analysis. On the other hand, a higher relative frequency of ADRs to AMPs from injectables than other dosage forms was found in this study as well as in EvaMed.

\subsection{Implications for Further Research}

Suspected adverse reactions to medicinal products authorized in the European Economic Area, including ADRs related to AMPs, are collected in the EudraVigilance database. Since 2011, the number of research publications using EudraVigilance data has been constantly increasing [49]. Instead of retrieving pharmacovigilance data from MAHs, as in the present study, future studies on the safety of AMP therapy may consider using data from the EudraVigilance database and including data from AMPs sold and used all over Europe. Furthermore, it is desirable to update previous systematic reviews on AMP safety in clinical studies [19, 23].

\section{Conclusions}

Retrolective analysis of pharmacovigilance data from spontaneous reporting systems is an important method to investigate and monitor the safety of AMP therapy in everyday practice. In the present study, ADRs related to AMP therapy were very rare and, as in previously published studies, mostly reversible and non-serious in nature. This applied to the whole dataset and all subgroups analysed. Notably, the frequency of ADRs in this study based on spontaneous reporting (1.5 ADR per million MDADs) is not directly comparable to frequencies in prospective clinical studies nor to frequencies based on other measures of patient exposure than MDADs, such as numbers of users or prescriptions.

Supplementary Information The online version contains supplementary material available at https://doi.org/10.1007/s40801-021-00262-7.

Acknowledgements This analysis and publication were commissioned by the European Scientific Cooperative on Anthroposophic Medicinal Products (ESCAMP: http://www.escamp.org), and performed with financial support from two foundations: Iona Stichting, Amsterdam, NL and Software AG-Stiftung, Darmstadt, Germany.

\section{Declarations}

Funding Financial support was received from two foundations, Iona Stichting (Amsterdam, The Netherlands) and Software AG-Stiftung (Darmstadt, Germany).

Conflict of interest ESCAMP has received funding from the AMP MAHs WALA Heilmittel GmbH and Weleda AG that provided data for this study. In accordance with the conflict-of-interest policy of ESCAMP (http://www.escamp.org/conflict-of-interests.html), sponsors had no influence on the planning or conduct of this study; the collection, preparation, analysis or interpretation of data for this article; nor on the preparation, review or approval of the article.

Ethics statement According to the Ethics Committee in Brabant, The Netherlands (no. 18.430, 2018-10-10) this study did not involve experiments with patients or study subjects, according to the Dutch Medical Research in Human Subjects Act (WMO). Therefore, the study was exempt from further medical ethics review. The study protocol was registered in the Dutch Trial Database on 24 September 2018 (http:// www.trialregister.nl, NTR no. 7628).

Consent to participate MAHs provided written informed consent to participate in the study.

Consent for publication MAHs provided written informed consent for the results of the data analysis to be published.

Availability of data and material Raw data are non-public data obtained from WALA Heilmittel GmbH, Weleda AG, Helixor Heilmittel GmbH, and Iscador AG for the purposes of this study and the presentation of the results in the form of the tables presented in the publication.

Code availability SPSS code used for the data analysis can be requested from the corresponding author. 
Author contributions All authors were involved in the development of the study design and approved the study protocol. MJ was study coordinator, co-writer of the study protocol and statistical analysis plan, cross-checked data-entry and coding, calculated MDADs sold, interpreted study results, and wrote the manuscript. HvW calculated MDADs sold, performed statistical analyses and interpreted study results. AG entered, coded and prepared the data for analysis. EB was co-writer of the study protocol. HJH was co-writer of the study protocol and statistical analysis plan, interpreted study results and co-wrote the final manuscript. All authors read, commented on and approved the final manuscript.

Open Access This article is licensed under a Creative Commons Attribution-NonCommercial 4.0 International License, which permits any non-commercial use, sharing, adaptation, distribution and reproduction in any medium or format, as long as you give appropriate credit to the original author(s) and the source, provide a link to the Creative Commons licence, and indicate if changes were made. The images or other third party material in this article are included in the article's Creative Commons licence, unless indicated otherwise in a credit line to the material. If material is not included in the article's Creative Commons licence and your intended use is not permitted by statutory regulation or exceeds the permitted use, you will need to obtain permission directly from the copyright holder. To view a copy of this licence, visit http://creativecommons.org/licenses/by-nc/4.0/.

\section{References}

1. Baars EW, Hamre HJ. Whole medical systems versus the system of conventional biomedicine: a critical, narrative review of similarities, differences, and factors that promote the integration process. Evid Based Complement Altern Med. 2017. https://doi. org/10.1155/2017/4904930.

2. Kienle GS, Albonico HU, Baars E, Hamre HJ, Zimmermann P, Kiene H. Anthroposophic medicine: an integrative medical system originating in Europe. Glob Adv Health Med. 2013. https://doi. org/10.7453/gahmj.2012.087.

3. The system of anthroposophic medicine. Brussels: International Federation of Anthroposophic Medical Associations (IVAA); 2014. https://www.ivaa.info/fileadmin/user_upload/pdf/broch ures_and_books/The_system_of_Anthroposophic_Medicine_ 2014.pdf. Accessed 7 June 2021

4. Mennet-von Eiff M, Greco A, Jones G, Kaltenbach M, Pedersen P, Rohner C, Schwarz R, editors. Anthroposophic pharmaceutical codex APC, edition 4.2. Dornach: International Association of Anthroposophic Pharmacists; 2020. https://iaap-pharma.org/ fileadmin/user_upload/pdf/apc/Anthroposophic_Pharmaceutical_ Codex_APC_edition_4.2.pdf. Accessed 4 May 2021.

5. Medicinal Products Act (Arzneimittelgesetz-AMG). Gesetz über den Verkehr mit Arzneimitteln. Art. 4, (33). Bundesministeriums der Justiz und für Verbraucherschutz; 2005. http://www.gesetzeim-internet.de/englisch_amg/. Accessed 4 May 2021.

6. Law of the Swiss Institute of Medicinal Products concerning the simplified Authorisation of Complementary and Phyto Medicines (Verordnung des Schweizerischen Heilmittelinstituts über die vereinfachte Zulassung von Komplementär- und Phytoarzneimitteln). Art. 4, 2 f. Swissmedic; 2018. https://www.admin.ch/opc/ de/classified-compilation/20173469/201901010000/812.212.24. pdf. Accessed 4 May 2021.

7. Hamre HJ, Glockmann A, Heckenbach K, Matthes H. Use and safety of anthroposophic medicinal products: an analysis of 44,662 patients from the EvaMed Pharmacovigilance Network.
Drugs Real World Outcomes. 2017. https://doi.org/10.1007/ s40801-017-0118-5.

8. Safety issues in the preparation of homeopathic medicines. Geneva: World Health Organization; 2009. https://apps.who.int/ iris/bitstream/handle/10665/44238/9789241598842_eng.pdf? sequence $=1 \&$ isAllowed=y. Accessed 4 May 2021 .

9. Buchholzer ML, Werner C, Knoess W. Current concepts on integrative safety assessment of active substances of botanical, mineral or chemical origin in homeopathic medicinal products within the European regulatory framework. Regul Toxicol Pharmacol. 2013. https://doi.org/10.1016/j.yrtph.2013.12.008.

10. Mol C, Roemer F, Scheer R, et al. Arzneimittel [überarbeitete Monographien der Kommission C]. In: Glöckler M, editor. Anthroposophische Arzneitherapie für Ärzte und Apotheker. Stuttgart: Wissenschaftliche Verlagsgesellschaft; 2008.

11. Complementary and alternative medicines (CAM) and traditional medicinal products (TMP). Bonn: Federal Institute for Drugs and Medical Devices (BfArM); 2013. https://www.bfarm.de/EN/Drugs/ licensing/zulassungsarten/pts/_node.html. Accessed 2 Mar 2021.

12. Pharmacovigilance. Bonn: Federal Institute for Drugs and Medical Devices (BfArM); 2013. https://www.bfarm.de/EN/Drugs/ vigilance/_node.html. Accessed 9 Mar 2021.

13. Jeschke E, Ostermann T, Tabali M, Kroz M, Bockelbrink A, Witt CM, et al. Anthroposophic medicine in pediatric primary care: a prospective, multicenter observational study on prescribing patterns. Altern Ther Health Med. 2011;17(2):18-28.

14. IVAA. Facts and figures on Anthroposophic Medicine (AM) in Europe and worldwide; 2012. http://www.antroposofinenlaaketiede. fi/pdf/Facts_and_Figures_World_Internet.pdf. Accessed 4 May 2021.

15. Arendt A, Debus M, Gutsch J, Karutz M, Kuck A, Kummer K-R, et al. Vademecum of Anthroposophic Medicines. Best practices for mistletoe use in cancer care. Der Merkurstab. 2019;72(Supplement):1-218.

16. Tröger W, Galun D, Reif M, Schumann A, Stankovic N, Milicevic M. Viscum album [L.] extract therapy in patients with locally advanced or metastatic pancreatic cancer: a randomised clinical trial on overall survival. Eur J Cancer. 2013. https://doi. org/10.1016/j.ejca.2013.06.043.

17. Loef M, Walach H. Quality of life in cancer patients treated with mistletoe: a systematic review and meta-analysis. BMC Complement Med Ther. 2020. https://doi.org/10.1186/ s12906-020-03013-3.

18. Heinrich M, Barnes J, Gibbons S, Williamson E. Fundamentals of pharmacognosy and phytotherapy. London: Churchill Livingstone; 2012.

19. Kienle GS, Glockmann A, Grugel R, Hamre HJ, Kiene H. Clinical research on anthroposophic medicine: update of a health technology assessment report and status quo. Forsch Komplementmed. 2011. https://doi.org/10.1159/000331812.

20. Kienle GS, Ben-Arye E, Berger B, Cuadrado Nahum C, Falkenberg T, Kapócs G, Kiene H, Martin D, Wolf U, Szöke H. Contributing to global health: development of a consensus-based whole systems research strategy for Anthroposophic Medicine. Evid Based Complement Altern Med. 2019. https://doi.org/10. 1155/2019/3706143

21. Hamre HJ, Glockmann A, Fischer M, Riley DS, Baars E, Kiene $\mathrm{H}$. Use and safety of anthroposophic medications for acute respiratory and ear infections: a prospective cohort study. Drug Target Insights. 2007;2:209-19.

22. Hamre HJ, Witt CM, Glockmann A, Troger W, Willich SN, Kiene H. Use and safety of anthroposophic medications in chronic disease: a 2-year prospective analysis. Drug Saf. 2006. https://doi.org/10.2165/00002018-200629120-00008.

23. Kienle GS, Kiene H, Albonico HU. Anthroposophic medicine: effectiveness, utility, costs, safety. Stuttgart: Schattauer Verlag; 2006.

24. Steele ML, Axtner J, Happe A, Kroz M, Matthes H, Schad F. Adverse drug reactions and expected effects to therapy with 
subcutaneous mistletoe extracts (Viscum album L.) in cancer patients. Evid Based Complement Alternat Med. 2014. https:// doi.org/10.1155/2014/724258.

25. Süsskind M, Thürmann PA, Lüke C, Jeschke E, Tabali M, Matthes $\mathrm{H}$, et al. Adverse drug reactions in a complementary medicine hospital: a prospective, intensified surveillance study. Evid Based Complement Altern Med. 2012. https://doi.org/10.1155/ 2012/320760.

26. Jong MC, Jong MU, Baars EW. Adverse drug reactions to anthroposophic and homeopathic solutions for injection: a systematic evaluation of German pharmacovigilance databases. Pharmacoepidemiol Drug Saf. 2012. https://doi.org/10.1002/pds.3298.

27. Regulation (EU) No 1235/2010 of the European Parliament and of the Council, amending, as regards pharmacovigilance of medicinal products for human use, Regulation (EC) No 726/2004 laying down Community procedures for the authorisation and supervision of medicinal products for human and veterinary use and establishing a European Medicines Agency, and Regulation (EC) No 1394/2007 on advanced therapy medicinal products. OJEU; 2010. https://eur-lex.europa.eu/LexUriServ/LexUriServ.do?uri= OJ:L:2010:348:0001:0016:EN:PDF. Accessed 4 May 2021.

28. Directive 2010/84/EU of the European Parliament and of the Council, amending, as regards pharmacovigilance, Directive 2001/83/EC on the Community code relating to medicinal products for human use. OJEU; 2010. https://eur-lex.europa.eu/LexUr iServ/LexUriServ.do?uri=OJ:L:2010:348:0074:0099:EN:PDF. Accessed 4 May 2021.

29. Guideline on good pharmacovigilance practices (GVP). Module I-pharmacovigilance systems and their quality systems. EMA/541760/2011 European Medicines Agency; 2012. https:// www.ema.europa.eu/en/documents/scientific-guideline/guidelinegood-pharmacovigilance-practices-module-i-pharmacovigilancesystems-their-quality-systems_en.pdf. Accessed 4 May 2021.

30. Commission Implementing Regulation (EU) No 520/2012 on the performance of pharmacovigilance activities provided for in Regulation (EC) No 726/2004 of the European Parliament and of the Council and Directive 2001/83/EC of the European Parliament and of the Council. OJEU; 2012. https://eur-lex.europa.eu/LexUriServ/LexUriServ.do? uri=OJ:L:2012:159:0005:0025:EN:PDF. Accessed 4 May 2021.

31. ICH Harmonised Tripartite Guideline. Maintenance of the ICH Guideline on Clinical Safety Data Management: data elements for transmission of individual case safety reports E2B(R2) International Conference on Harmonisation (ICH); 2001. https://admin. ich.org/sites/default/files/inline-files/E2B_R2_Guideline.pdf. Accessed 4 May 2021.

32. Guideline on good pharmacovigilance practices (GVP). Productor population-specific considerations IV: paediatric population. European Medicines Agency (EMA); 2018. https://www.ema. europa.eu/en/documents/scientific-guideline/guideline-goodpharmacovigilance-practices-gvp-product-population-specificconsiderations-iv_en-0.pdf. Accessed 4 May 2021.

33. Safety monitoring of medicinal products: guidelines for setting up and running a pharmacovigilance centre. Uppsala: Uppsala Monitoring Centre; 2000. http://digicollection.org/hss/en/d/Jh293 4e/. Accessed 4 May 2021.

34. Brown EG, Wood L, Wood S. The medical dictionary for regulatory activities (MedDRA). Drug Saf. 1999. https://doi.org/10. 2165/00002018-199920020-00002.

35. Frequency of adverse drug reactions (CIOMS). In: Definitions. Geneva: World Health Organization; 2007. p. 10. http://www. who.int/medicines/areas/quality_safety/safety_efficacy/trainingco urses/definitions.pdf. Accessed 4 May 2021.
36. Regulation (EU) 2016/679 of the European Parliament and of the Council and Directive on the protection of natural persons with regard to the processing of personal data and on the free movement of such data, and repealing Directive 95/46/EC (General Data Protection Regulation). OJEU; 2016. https://eur-lex.europa. eu/eli/reg/2016/679/oj. Accessed 4 May 2021.

37. Teschke R, Eickhoff A, Schulze J, Danan G. Herb-induced liver injury (HILI) with 12,068 worldwide cases published with causality assessments by Roussel Uclaf Causality Assessment Method (RUCAM): an overview. Transl Gastroenterol Hepatol. 2020. https://doi.org/10.21037/tgh-20-149.

38. Danan G, Teschke R. RUCAM in drug and herb induced liver injury: the update. Int J Mol Sci. 2016. https://doi.org/10.3390/ ijms 17010014.

39. Teschke R, Frenzel C, Glass X, Schulze J, Eickhoff A. Greater Celandine hepatotoxicity: a clinical review. Ann Hepatol. 2012. https://doi.org/10.1016/S1665-2681(19)31408-5.

40. Pantano F, Mannocchi G, Marinelli E, et al. Hepatotoxicity induced by greater celandine (Chelidonium majus L.): a review of the literature. Eur Rev Med Pharmacol Sci. 2017;21(1 Suppl):46-52.

41. Thiele A. Abwehr von Gefahren durch Arzneimittel, Stufe II hier: Schöllkraut-haltige Arzneimittel zur innerlichen Anwendung. 75-3822-A-10488-98297/08. Bonn: Bundesinstitut für Arzneimittel und Medizinprodukte; 2008. https://www.bfarm.de/Share dDocs/Downloads/DE/Arzneimittel/Pharmakovigilanz/Risikoinfo rmationen/RisikoBewVerf/s-z/schoellkraut_bescheid_080409. pdf?_blob=publicationFile\&v=2. Accessed 4 May 2021.

42. Teschke R, Glass X, Schulze J. Herbal hepatotoxicity by Greater Celandine (Chelidonium majus): causality assessment of 22 spontaneous reports. Regul Toxicol Pharmacol. 2011. https://doi.org/ 10.1016/j.yrtph.2011.08.008.

43. Teschke R, Danan G. Idiosyncratic Drug-Induced Liver Injury (iDILI) and Herb-Induced Liver Injury (HILI): diagnostic algorithm based on the Quantitative Roussel Uclaf Causality Assessment Method (RUCAM). Diagnostics. 2021. https://doi.org/10. 3390/diagnostics11030458.

44. Gonzalez-Gonzalez C, Lopez-Gonzalez E, Herdeiro MT, Figueiras A. Strategies to improve adverse drug reaction reporting: a critical and systematic review. Drug Saf. 2013. https://doi.org/10. 1007/s40264-013-0058-2.

45. Hazell L, Shakir SA. Under-reporting of adverse drug reactions: a systematic review. Drug Saf. 2006. https://doi.org/10.2165/00002 018-200629050-00003.

46. Shaw D, Ladds G, Duez P, Williamson E, Chan K. Pharmacovigilance of herbal medicine. J Ethnopharmacol. 2012. https://doi.org/ 10.1016/j.jep.2012.01.051.

47. Svedlund E, Larsson M, Hägerkvist R. Spontaneously reported adverse reactions for herbal medicinal products and natural remedies in Sweden 2007-15: report from the Medical Products Agency. Drugs Real World Outcomes. 2017. https://doi.org/10. 1007/s40801-017-0104-y.

48. Eudralex Volume 9A of The Rules Governing Medicinal Products in the European Union. Guidelines on pharmacovigilance for medicinal products for human use. 2008. https://ec.europa.eu/ health/sites/health/files/files/eudralex/vol-9/pdf/vol9a_09-2008_ en.pdf. Accessed 4 May 2021.

49. Postigo R, Brosch S, Slattery J, van Haren A, Dogné J-M, Kurz X, et al. EudraVigilance Medicines Safety Database: publicly accessible data for research and public health protection. Drug Saf. 2018. https://doi.org/10.1007/s40264-018-0647-1. 